\title{
Cross-Sectional Area Estimation of hot rolled round steel bars using eddy currents
}

\author{
Jens Weidenmüller ${ }^{1}$, Christoph Knopf ${ }^{1}$, Christian Sehestedt ${ }^{1}$, Olfa Kanoun ${ }^{2}$, Jörg Himmel ${ }^{1}$ \\ ${ }^{1}$ Laboratory for Sensor Technology and Measurement Engineering \\ University of Applied Sciences Koblenz, RheinAhrCampus 53424 Remagen, Germany \\ e-mail: weidenmueller@rheinahrcampus.de, sehestedt@rheinahrcampus.de, \\ cknopf@rheinahrcampus.de, himmel@rheinahrcampus.de \\ ${ }^{2}$ Department for Electrical Engineering and Information Technology \\ Technische Universität Chemnitz, 09111 Chemnitz, Germany \\ kanoun@ieee.org
}

\begin{abstract}
The cross-sectional area is an important quality parameter of hot rolled round steel bars which at best is determined during the production process. Therefore, robust non-contact measurement systems are necessary which are applicable even in difficult environmental conditions as occurring in steel plants. Currently, measurement systems based on optical sensors are used in this measuring task. Integrating such optical systems into places under harsh conditions, such as steam, moistening of the rod with drilling or cutting fluid and high temperatures above the Curie point, cause a lot of effort and the measurement inaccuracy increases rapidly. This paper presents a measurement system with a novel transducer arrangement based on high frequency eddy currents which are less effected by those interferences.
\end{abstract}

\section{Introduction}

According to DIN EN 10060:2003, the quality of hot rolled round steel bars is basically divided into two tolerance classes, normal and precision. In order to figure out whether the produced steel bar meets the requirements for general or precision purpose the rod's cross-sectional area must be determined. At best, this parameter should be estimated directly in the production process as the rolls and the rod's velocity in the production line can be readjusted immediately. In this way, the produced round steel bars have a high standard of quality with reduced rejections.

The main challenges in this measurement application are the high speed of the rods up to $70 \mathrm{~m} / \mathrm{s}$, high temperatures above the Curie point and the difficult conditions in steel plants. Furthermore, a non-contact measurement is required as a touching point could damage either the detection unit or the steel bars. Based on the experiences of the material tracking sensor (Fig.1a), which was developed earlier in the Laboratory of Sensor Technology and Optical Measurement (LASOM) and successfully tested in the steel plant Siegen EWS (Fig.1b), a new sensor with the promising high frequency eddy current technology is being investigated [8].

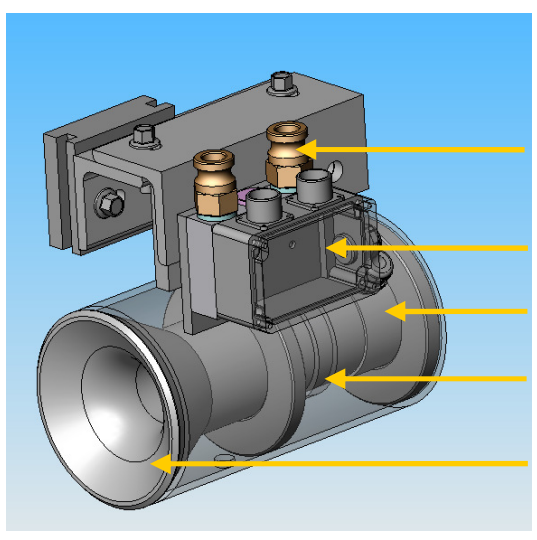

a) SolidWorks ${ }^{(\mathcal{)}}$ engineering drawing
Cooling System

Electronics Box

Ceramic

Coil

Guideway

Fig. 1 Material Tracking Sensor

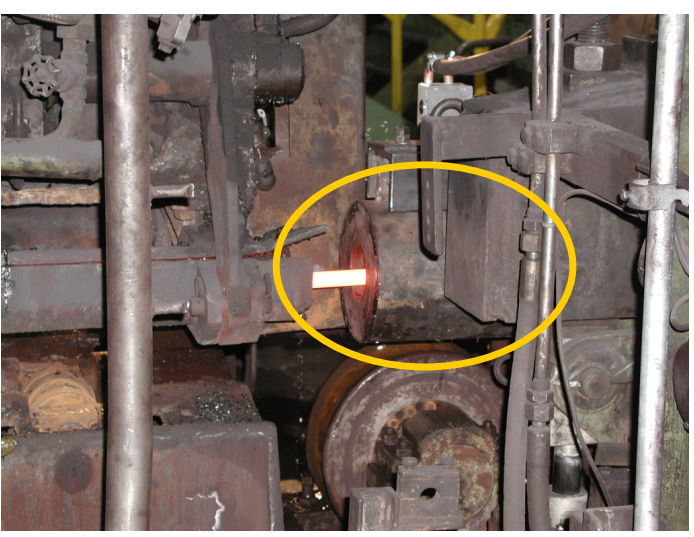

b) Sensor into the production process DEW steel plant 
Basically, the material tracking sensor consists of a detection coil, which is placed symetrically around the measuring section, and a capacitor that tunes the parallel RLC oscillator to the desired frequency of about $13 \mathrm{MHz}$. If the steel bar enters the measuring section, the inductance $L_{C}$ of the detection coil is coupled with the mutual inductance $L_{M}$ caused by the circular eddy currents on the rod surface. In this setup, the resulting inductance $L_{\sigma}$ is detected as a varying frequency of the active RLC oscillator. The theory of this measuring principle is described in earlier papers using the so-called multi-transformer model[1][5].

This arrangement is suitable for material tracking. However, initial setups indicate that a more extensive data set is required for cross-sectional area estimation. Therefore a measurement system that also provides information about the complex impedance spectrum near the resonant frequency was developed for this new application. More precisely, the active RLC oscillator is replaced by a High Frequency Vector Network Analyzer (HF VNA) based on the reflection measurement. This HF VNA detects the impedance spectrum of the passive RLC oscillator. Aditionally, the sensor was redesigned resulting in a novel measurement setup with three detection coils arranged in an angle of $120^{\circ}$ (Fig.3b). This add-on is nessesary as the measured impedance spectrum depends not only on the cross-sectional area of the rod but also on the rod position and surrounding temperature. Using those linearly independent results of the three detection units the rod's cross-sectional area can be estimated.[4][6][7]

\section{Sensor Design}

The engineering drawing of the assembled sensor is shown in figure $3 a$. The complete system is mounted on the production line via quick clamp device. This design is necessary to make the sensor easily replaceable in case of errors and guarantees the needed space for maintenance. Another important design feature is the guidance at the sensor front, as the material flow may under no circumstances be affected by the measurement system. Additionally, the shielding layers, which are primarily necessary to decouple the electromagnetic fields of the three detection coils from each other, are also conically designed to guide the steel bars a further two times inside the sensor. Finally, the three detection coils are fixed using a fibre ceramic substrate. A fixed coil position is essential to estimate the cross sectional area from the recorded look-up table that contains a set of characteristic curves for each rod diameter.

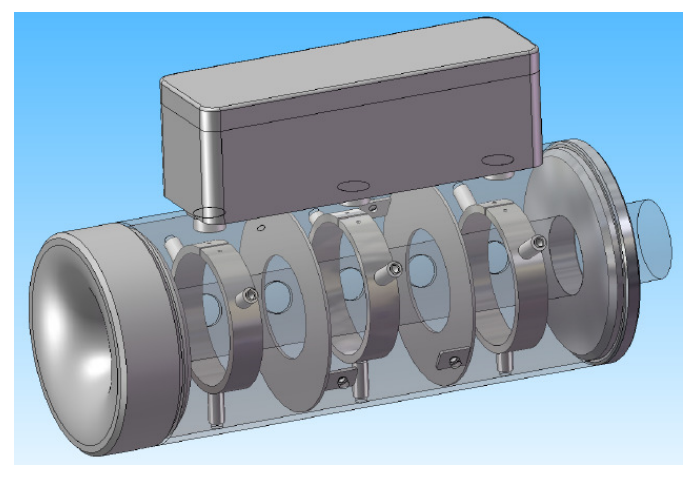

a) SolidWorks ${ }^{(}$engineering drawing

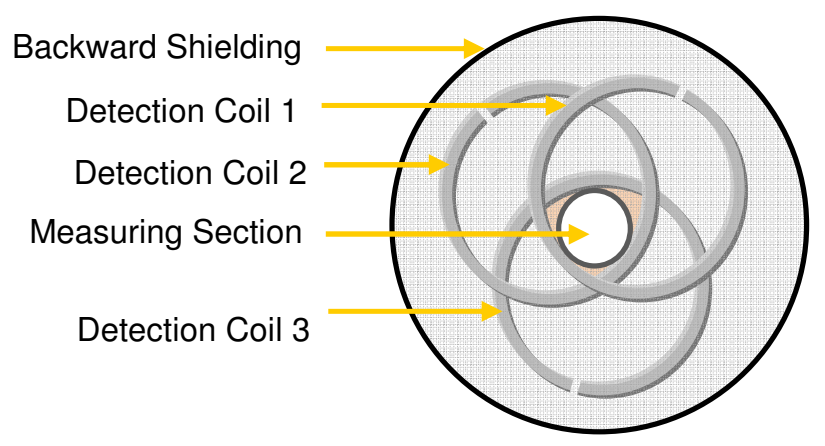

b) Front View of the $120^{\circ}$ detection coil arrangment

Fig. 3 Cross-Sectional Area Estimation

\section{HF Vector Network Analyzer}

The measurement part of the HF VNA, including source, bridge and detector, is placed in a waterproof and temperature controlled box outside of the backward shielding as show in figure 3a. The control part, which drives the signal source and provides the data collection and processing part, is situated in a PC. Amongst others, that makes the HF VNA cost efficient and suitable for applications close to hot rolled steel bars. The block diagram of the HF VNA is displayed in figure 4. The complex impedance spectrum of the RLC oscillator is obtained in typical VNA usage. The RF DDS provides the sinusoidal signal to the bridge during the reflection measurements. The reflection bridge acts as terminal for the Device Under 
Test (DUT) as well as for the open, short and load calibration standards and is necessary to separate the incident from the reflected signal. Unlike in the typical HF VNA mode of operation, the OSL calibration standards cannot be applied to the reflection bridge manually, as during manufacturing the area around the sensor is a locked safety zone. Hence, the HF VNA must be automatically calibrated. That is achieved using a microcontroller based switching system. Due to the good HF characteristics reed relays are implemented to separate the desired signal.

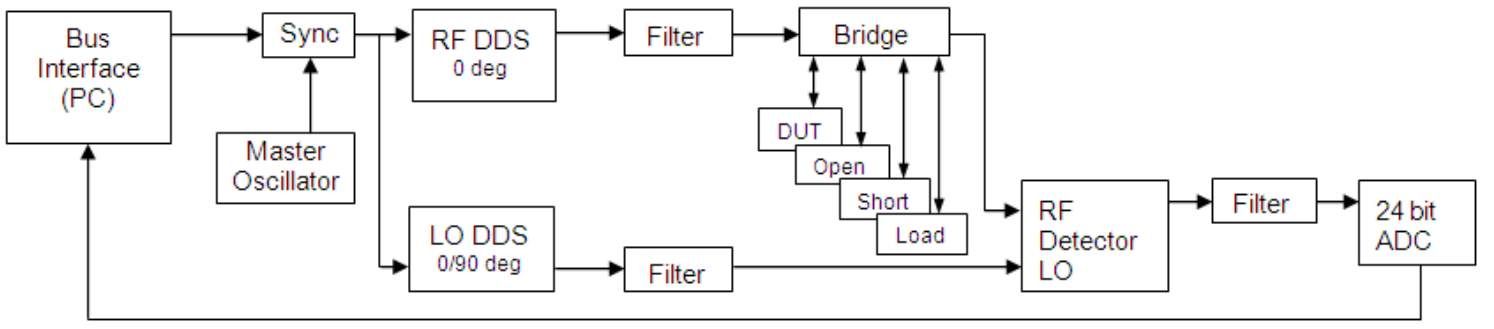

Fig. 4 VNA Block Diagram

The LO DDS is frequency and phase synchronized with the RF DDS in order to provide the reference signal which is needed in the detection part. The RF DDS is always programmed for 0 degrees while the LO DDS is typically programmed for either 0 or 90 degrees. The VNA detector is realized using a linearized Gilbert cell mixer, analog filtering and buffering and a precise 24-bit ADC. As both DDSs are programmed with the same frequency, the voltage at the detector output is DC and contains the desired carrier information. As the phase of the LO DDS can be programmed to values of 0 and 90 degrees, the detector output takes on two DC values that together represent the vector components of the applied RF signal. Following from those results combined with the OSL calibration data the complex impedance spectrum is obtained. The main parts, which are the signal source, bridge, detector and also the profound arithmetic, are described in the paper "Rod Shape Testing by High Frequency Eddy Current -Passive Impedance Measurement-" [2]. The key facts of the signal source and the detector are listed in table 1.

\begin{tabular}{|c|c|c|c|c|}
\hline Description & min & value & $\max$ & unit \\
\hline \multicolumn{5}{|l|}{ Signal Source } \\
\hline Frequency Range & 0.05 & & 60 & $\mathrm{MHz}$ \\
\hline Frequency Resolution & & 0.035 & & $\mathrm{~Hz}$ \\
\hline Master Oscillator & & 148.344 & & $\mathrm{MHz}$ \\
\hline Phase Range & 0 & 11.25 (steps) & 360 & Degree \\
\hline \multicolumn{5}{|l|}{ Detector } \\
\hline A/D Converter Resolution & & 0.149 & & $\mu \mathrm{V}$ \\
\hline A/D Conversion Speed & 7 & & 3500 & $\mathrm{~Hz}$ \\
\hline \multicolumn{5}{|l|}{ Reflection Bridge } \\
\hline Matched Impedance & & 50 & & Ohm \\
\hline Static Contact Resistance (reed relays) & & 250 & & $\mathrm{mOhm}$ \\
\hline Insulation Resistance (reed relays) & $10^{12}$ & $10^{13}$ & & Ohm \\
\hline Capacitance (reed relays) & & 0.1 & & $\mathrm{pF}$ \\
\hline
\end{tabular}

Tab. 1 HF VNA Key Data

\section{Laboratory Setup}

Basically, the laboratory setup was developed for attainment of two aims. First, the results of the purposebuilt HF VNA need to be compared to a laboratory quality Impedance Analyzer, in this case an Agilent 4294A, and second to gain calibration data for different rod shapes and positions. Those calibration data are essential as the rod's cross-sectional area cannot be calculated from the measured impedance spectrum of each detection unit directly.

Figure 5 shows the block diagram of the laboratory setup. Using this setup, different steel bars can be easily attached to the software controlled 2D positioning device. The maximum travel of the step motors is $50 \mathrm{~mm}$ with a drive dependent resolution, in this case $1 \mu \mathrm{m}$. The impedance spectrum of the detection units can be obtained with the HF VNA reflection measurement firstly, as shown in figure 5 , and secondly with the impedance analyzer Agilent 4294A. Hence, the results of both impedance measurement systems in the expected range between 10 and $200 \mathrm{ohm}$ can be compared exactly. Additionally, as mentioned in the introduction part, temperature effects need to be considered as well. Therefore, the setup is placed in a climatic test chamber in order to gain adjustable and stabile temperature and humidity conditions. 


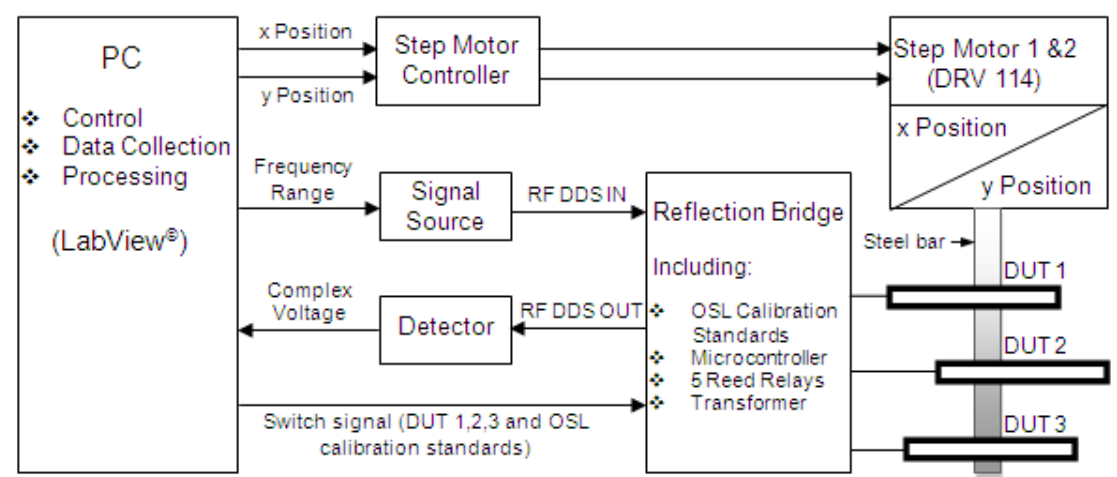

Fig. 5 Block Diagram of the Laboratory Setup

\section{First Test Results}

In order to gain better understanding figure 5 displays the position dependent absolute value impedance $|\mathrm{Z}|$ examplarly for one RLC-oscillator. The rod is moved inside one detection coil using the described laboratory setup. The parameters of interest in that arrangement are listed in table 2.

\begin{tabular}{lcc}
\hline \multicolumn{1}{c}{ Parameter } & value & Unit \\
\hline Detection Coil & & $\mathrm{mm}$ \\
Inner Diameter & 51 & $\mathrm{~mm}$ \\
Outer Diameter & 59 & \\
Winding & 1 & $\mathrm{~mm}$ \\
Rod & 3 & $\mathrm{~mm}$ \\
$\quad$ Clearance Distance (Coil-Rod) & 10 & $\mathrm{~mm}$ \\
Rod Diameter & 1 & ${ }^{\circ} \mathrm{C}$ \\
\hline Step Size (Step Motors) & 25 & \\
\hline Temperature & & \\
\hline
\end{tabular}

Tab. 2 Setup Parameter

Figure 6a shows the RLC oscillator's absolute value impedance $|\mathrm{Z}|$ detected with the Agilent 4294A whereas figure $6 b$ shows the results of the same setup measured with the purpose-built HF VNA. Basically, both measurement systems display similar performance. Anyhow, the data acquisition speed of the HF VNA, unlike the Agilent 4294A's, is matched to the task. The ADC conversion time is set to the fastest performance of $3.5 \mathrm{kHz}$ to support process control. The inductance of the detection coil in use is about $240 \mathrm{nH}$ and the passive RLC oscillator is tuned to the desired resonant frequency of $13 \mathrm{MHz}$.

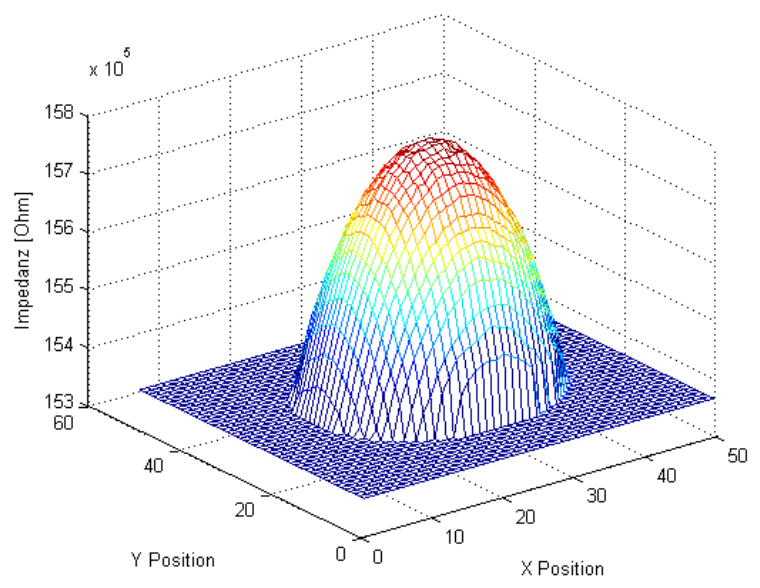

a) Agilent 4294A

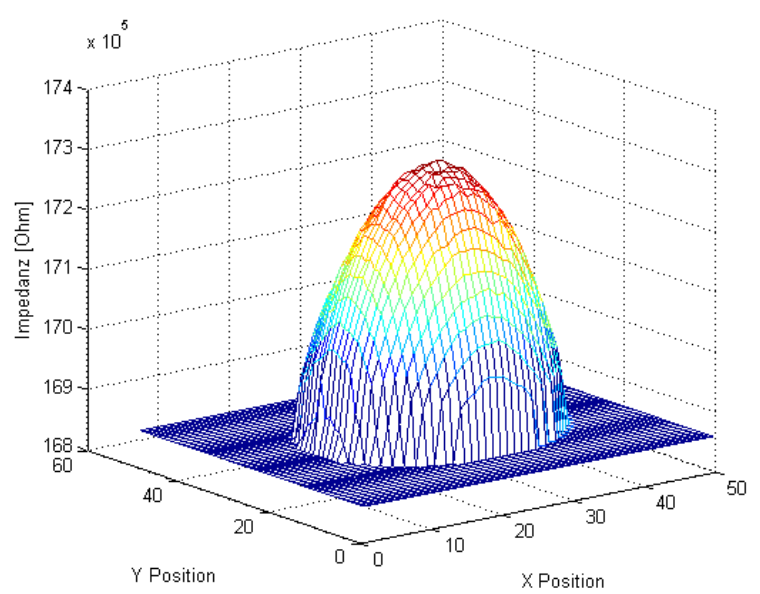

b) HF VNA

Fig. 6 Absolute Value Impedance $|\mathrm{Z}|$ at $12.287,500 \mathrm{MHz}$ (the area surrounding the measuring section is set to lowest measured impedance to gain high plot dynamic) 
As a result, the expected impedance characteristics can be gathered from both diagrams. If the rod is situated at centre position the absolute value impedance $|Z|$ peaks and drops for decreasing distances between the rod and detection coil. The frequency sweep ranges from 10 to $15 \mathrm{MHz}$ in order to gain the oscillator's resonance curves which are typic for each position. From this set of characteristic resonance curves it follows that the highest change of impedance (approximately $4 \mathrm{ohm}$ ) is achieved at $12.287,500$ $\mathrm{MHz}$. In the next step, the impedance characteristics of rod diameters that need to be considered will be recorded at the desired frequency of $12.287,500 \mathrm{MHz}$. This set of characteristic curves for each rod diameter will be saved in a look-up table and the cross-sectional area can be estimated from the measured three results.

\section{Conclusion}

The new high frequency eddy current cross-sectional area estimation has several advantages compared to sensor systems currently in use. The promising test results using the laboratory setup (Fig.5) will be verified in a steel plant as the designed HF VNA is applicable into places with harsh environmental conditions. Due to the compact and robust front-end electronics the HF VNA can be located close to the detection unit which is necessary in order to gain stable measurement conditions. Also the data acquisition speed is sufficient to support process control. Additionally, according to the experiences of the material tracking application an operational sensor design was developed. Using the explained laboratory setup the impedance characteristics of all considered rod diameters can be measured and saved in a look-up table. Finally, referring to the obtained database a mathematical approach will be developed to estimate the cross-sectional area of the rod [3].

\section{References}

[1] Weidenmüller J., Knopf C., Sehestedt C., Himmel J., Rod Shape Testing by High Frequency Eddy Current - The experimental setup, I'MTC 2008 Conference IEEE IM Chapter, 14.05.2008 Vancouver Island,Verlag: IEEE Catalog Number: 08CH37941C Seite 1482-1486,

[2] Weidenmüller J., Sehestedt C., Kanoun O., Himmel J., Rod Shape Testing by High Frequency Eddy Current -Passive Impedance Measurement-, SSD 2006 International Multi-Conference on Systems, Signal and Devices, 23.03.2009 Djerba, Accepted Paper

[3] Liu G., Li Y., Sun Y., Sacks P., Udpa S., An iterativ algorithm for eddy current signals inversion, AlP Conference Proceedings, Band 509 A (2000) Seite 497-504.

[4] Simonyi K. Theoretische Elektrotechnik, VEB Deutscher Verlag der Wissenschaften, Edition 8 (1980) [5] Vyroubal D.: Impedance of the Eddy-Current Displacement Probe: The Transformer Model, IEEE Transactions on Instrumentation and Measurement, Vol. 53, No. 2, April 2004.

[6] Philippow E., Grundlagen der Elektrotechnik, Verlag Technik, Edition 10 (2000)

[7] Zinke O., Brunswig H., Hochfrequenztechnik 1- Hochfrequenzfilter, Leitungen, Antennen, Springer Verlag, Edition 6 (2000)

[8] Himmel J., Arend S., Otto A., Sehestedt Ch., Produktionsflusskontrolle in Walzwerken mit elektromagnetischen Streufeldern, Sensoren Signale Systeme, Buchreihe Messen Prüfen Automatisieren, Bd. 6, b-Quadrat Verlag, Kreuztal 2006. 